\title{
Key features of the transient amplification of mistuned systems
}

\author{
Luigi Carassale \\ University of Genova, Genova, Italy \\ luigi.carassale@unige.it \\ Vincent Denoël \\ University of Liège, Liège, Belgium \\ v.denoel@uliege.be \\ Carlos Martel \\ Universidad Politécnica de Madrid, Madrid, Spain \\ carlos.martel@upm.es \\ Lars Panning-von Scheidt \\ Leibniz Universität Hannover, Hannover, Germany \\ panning@ids.uni-hannover.de
}

\begin{abstract}
The dynamic behavior of bladed disks in resonance crossing has been intensively investigated in the community of turbomachinery, addressing the attention to (1) the transient-type response that appear when the resonance is crossed with a finite sweep rate and (2) the localization of the vibration in the disk due to the blade mistuning. In real conditions, the two mentioned effects coexist and can interact in a complex manner. This paper investigates the problem by means of analytic solutions obtained through asymptotic expansions, as well as numerical simulations. The mechanical system is assumed as simple as possible: a 2-dof linear system defined through the three parameters: damping ratio $\xi$, frequency mistuning $\Delta$, rotor acceleration $\dot{\Omega}$. The analytic solutions are calculated through the multiple-scale method.
\end{abstract}

\section{INTRODUCTION}

The dynamic behavior of bladed disks in resonance crossing has been intensively investigated in the community of turbomachinery. In particular, the attention has been addressed towards two issues: (1) 
the transient-type response that appear when the resonance is crossed with a finite sweep rate and (2) the localization of the vibration in the disk due to the blade mistuning.

The transient effects produce a reduction of the maximum amplitude of the response and shift (upwards in run-up) the rotor speed at which the maximum response appears. Coherently with the common intuition, the reduction of the maximum amplitude of vibration is proportional to the rotor acceleration. That is, the faster the crossing of the resonance, the lower the response amplification e.g. [1-5].

Blade mistuning produces the increment of the dynamic response of some blades when the disk is excited with a resonant or quasi-resonant force. On this matter the literature is vast, and a wide list of references can be found for example in [6] and in the recent monography [7]. Analytical formulations developed in the quasi-steady regime, i.e. disregarding the transient effects due to the rotor acceleration, show that the increment of dynamic amplification compared to the tuned case is a function of the mistuning level and damping [8], as well as the number of active modes involved in the disk response [9].

In real conditions, the two mentioned effects coexist and can interact in a complex manner, e.g. [3, 10]. It was observed that the reduction of the dynamic response that is expected in resonant crossing due to the transient effects may vanish when dealing with mistuned disks [11]. For some systems it was even observed a weak over-amplification with respect to the quasi-steady prediction, which appears quite counterintuitive. This phenomenon has been called Transient Amplitude Amplification of Mistuned Systems (TAMS) and has been investigated both numerically and experimentally [12$14]$.

This paper investigates the TAMS by means of analytic solutions obtained through asymptotic expansions, as well as numerical simulations. The problem is studied working on the simplest possible bladed disk model able to produce TAMS, namely, a 2-dof linear system defined through the three parameters: damping ratio $\xi$, frequency mistuning $\Delta$, rotor acceleration $\dot{\Omega}$. The dynamic 
response is calculated using the multiple-scale method. The differential equation governing the evolution of the complex amplitude is derived both for the case of tuned and mistuned disks.

\section{IDEALIZED DISK MODEL}

In order to analyze the nature of the TAMS, we consider a mechanical system that, remaining as simple as possible, retains the important features of a bladed disk. To this purpose, we start from an idealized bladed disc composed by $N$ blades modeled as 1-dof systems. The equation of motion has the form

$$
\mathbf{M u}+\mathbf{K u}=\mathbf{f}
$$

where $\mathbf{M}$ and $\mathbf{K}$ are the mass and stiffness matrices, respectively, $\mathbf{u} \in \mathbb{R}^{N}$ is the displacement vector and $\mathbf{f} \in \mathbb{R}^{N}$ is the external force. The damping term is assumed to be small and will be introduced later.

\section{Tuned case}

If the disk is cyclic symmetric, $\mathbf{M}$ and $\mathbf{K}$ are circulant matrices, i.e. they have the structure [7]

$$
\mathbf{K}=\left[\begin{array}{ccccc}
k & k_{c} & & & k_{c} \\
k_{c} & k & k_{c} & & \\
& k_{c} & \ddots & \ddots & \\
& & \ddots & k & k_{c} \\
k_{c} & & & k_{c} & k
\end{array}\right]
$$

where $k$ and $k_{c}$ are, respectively, the direct blade stiffness and coupling stiffness between neighbor blades.

Under the cyclic symmetry condition, the vibration modes have harmonic shape, i.e. their $j^{\text {th }}$ components are in the form [7] 


$$
\begin{array}{ll}
\phi_{r, j}^{(c)}=\frac{1}{\sqrt{N}} \cos \left(2 \pi r \frac{j}{N}\right) \\
\phi_{r, j}^{(s)}=\frac{1}{\sqrt{N}} \sin \left(2 \pi r \frac{j}{N}\right)
\end{array} \quad\left(\begin{array}{l}
r=0 \ldots \text { floor }\left(\frac{N}{2}\right) \\
j=0 \ldots N-1
\end{array}\right)
$$

where $r$ is called harmonic index and floor $(\bullet)$ is the rounding towards $-\infty$. For the cases $r=0$ and $r=N / 2$ (if $N$ is even), the eigenvector $\phi_{r}^{(s)}$ does not exist. In the other cases, the eigenvectors $\phi_{r}^{(c)}$ and $\phi_{r}{ }^{(s)}$ share the same eigenvalue $\lambda_{r}=\omega_{r}^{2}$.

The force $\mathbf{f}$ is assumed as a traveling wave (TW) excitation, whose frequency changes linearly in time. It represents an Engine Order (EO) component of a synchronous excitation during a constantacceleration run-up or run-down. For reasons that will be clear afterwards, it is convenient to represent the force using the complex-valued notation

$$
\mathbf{f}=\boldsymbol{\psi}_{r} f \mathrm{e}^{\frac{1}{2} \mathrm{i} r \dot{\Omega} t^{2}}
$$

where $f$ is the force complex amplitude, the index $r$ is here interpreted as EO, $\dot{\Omega}$ is the rotor acceleration, referred to as chirp rate, and $\psi_{r}$ is a vector whose components $\psi_{r, j}$ are given as

$$
\psi_{r, j}=\frac{1}{\sqrt{N}} \mathrm{e}^{2 \pi \mathrm{i} r \frac{j}{N}} \quad(j=0, \ldots, N-1)
$$

Comparing Eq. (3) and Eq. (5), it can be deduced from the Euler's identity that

$$
\begin{aligned}
& \boldsymbol{\psi}_{r}=\phi_{r}^{(c)}+\mathrm{i} \phi_{r}^{(s)} \\
& \boldsymbol{\psi}_{-r}=\phi_{r}^{(c)}-\mathrm{i} \phi_{r}^{(s)}
\end{aligned}
$$

Since the vectors $\psi_{r}$ and $\psi_{-r}$ are given by two independent linear combinations of eigenvectors sharing the same eigenvalues, then they are eigenvectors as well. Due to their nature, $\phi_{r}{ }^{(c)}$ and $\phi_{r}{ }^{(s)}$ are called Standing Wave (SW) modes, while $\psi_{r}$ and $\psi-r$ are called Traveling Wave (TW) modes. 
Let us assume that the blade motion is entirely described by the mode pair with harmonic index $r$, i.e.:

$$
\begin{aligned}
\mathbf{u}(t) & =\operatorname{Re}\left(\phi_{r}^{(c)} x_{1}(t)+\phi_{r}^{(s)} x_{2}(t)\right) \\
& =\operatorname{Re}\left(\boldsymbol{\psi}_{r} y_{1}(t)+\boldsymbol{\psi}_{-r} y_{2}(t)\right)
\end{aligned}
$$

where the extraction of the real part is required due to the choice of using a complex-valued force in Eq. (4), thus $x_{j}$ and $y_{j}$ are complex valued as well.

The equations of motion projected on the SW modes read

$$
\begin{aligned}
& \ddot{x}_{1}+2 \xi \omega_{r} \dot{x}_{1}+\omega_{r}^{2} x_{1}=\phi_{r}^{(c)^{\mathrm{T}}} \mathbf{f}=f \mathrm{e}^{\frac{1}{2} \mathrm{i} r \dot{\Omega} t^{2}} \\
& \ddot{x}_{2}+2 \xi \omega_{r} \dot{x}_{2}+\omega_{r}^{2} x_{2}=\phi_{r}^{(s)^{\mathrm{T}}} \mathbf{f}=f \mathrm{i}^{\frac{1}{2} \mathrm{i} r \dot{\Omega} t^{2}}
\end{aligned}
$$

in which a viscous dissipation has been added and parametrized by the modal damping ratio $\xi$, assumed equal for both the modes.

The two Eqs. (8) are identical and are excited by equal forces with 90-degree phase shift. Besides, also the real and imaginary parts of both $x_{1}$ and $x_{2}$ have the same amplitude and 90-degree phase shift. If the equations of motion are projected on the TW modes, they assume the form

$$
\begin{aligned}
& \ddot{y}_{1}+2 \xi \omega_{r} \dot{y}_{1}+\omega_{r}^{2} y_{1}=\psi_{r}^{*} \mathbf{f}=f \mathrm{e}^{\frac{1}{2} \mathrm{i} r \dot{\Omega} t^{2}} \\
& \ddot{y}_{2}+2 \xi \omega_{r} \dot{y}_{2}+\omega_{r}^{2} y_{2}=\psi_{-r}^{*} \mathbf{f}=0
\end{aligned}
$$

where the superscript * represents the conjugate transpose. Also in this case the two equation of motion are identical, however the force appears only in the first equation, corresponding to the mode having the same whirl direction of the considered force. The relationship between SW and TW coordinates can be deduced by substituting Eqs. (6) into Eqs. (7) 


$$
y_{1}=\frac{x_{1}-\mathrm{i} x_{2}}{2} ; \quad y_{2}=\frac{x_{1}+\mathrm{i} x_{2}}{2}
$$

The assumption of having only two active modes is obviously a simplification with respect to the general case. However, it represents the realistic situation in which mistuning is small and the natural frequencies are well isolated [9].

\section{Mistuning}

Blade mistuning modifies the shape of the matrices $\mathbf{M}$ and $\mathbf{K}$ and, as a consequence, destroys the regular structure of eigenvalues and eigenvectors that have been described above [7].

Since we are interested in exploring simple systems, we assume that the blade vibration can be still represented by two vibration modes that retain the harmonic structure of the tuned disk. This happens rigorously if the two considered modes are isolated and the mistuning pattern has a harmonic shape with harmonic index $2 r$, i.e. the system matrices are updated as [9]:

$$
\begin{aligned}
& \mathbf{K} \leftarrow \mathbf{K}+\operatorname{diag}\left(\delta_{K} \boldsymbol{\psi}_{2 r}+\delta_{K}^{*} \boldsymbol{\psi}_{-2 r}\right) \\
& \mathbf{M} \leftarrow \mathbf{M}+\operatorname{diag}\left(\delta_{M} \boldsymbol{\psi}_{2 r}+\delta_{M}^{*} \boldsymbol{\psi}_{-2 r}\right)
\end{aligned}
$$

where $\delta_{K}$ and $\delta_{M}$ are complex-valued constants representing the amplitude of the mistuning and $\operatorname{diag}(\cdot)$ constructs a diagonal matrix from a vector. This result is actually obtained even when the mistuning pattern does not follow the mentioned condition, but, simply, when it is very small. As an effect of the mistuning, the double eigenvalue $\lambda_{r}$ splits into two separate eigenvalues $\lambda_{r, 1}=\omega_{r, 1}^{2}$ and $\lambda_{r, 2}=\omega_{r, 2}^{2}$. Due to this circumstance, the TW vectors $\psi_{r}$ and $\psi-r$ are not vibration modes of the system. Besides, the SW modes defined by Eq. (3) are modified as [15]:

$$
\begin{array}{ll}
\phi_{r, j}^{(c)}=\frac{1}{\sqrt{N}} \cos \left(2 \pi r \frac{j}{N}+\frac{\alpha}{2}\right) \\
\phi_{r, j}^{(s)}=\frac{1}{\sqrt{N}} \sin \left(2 \pi r \frac{j}{N}+\frac{\alpha}{2}\right)
\end{array} \quad\left(\begin{array}{l}
r=0 \ldots \text { floor }\left(\frac{N}{2}\right) \\
j=0 \ldots N-1
\end{array}\right)
$$


where the angle $\alpha$ depends on the constants $\delta_{K}$ and $\delta_{M}$ and is referred to as mode clocking.

The equations of motion in terms of SW coordinates are a simple update of Eqs. (8), i.e.,

$$
\begin{aligned}
& \ddot{x}_{1}+2 \xi(1-\Delta) \bar{\omega} \dot{x}_{1}+(1-\Delta)^{2} \bar{\omega}^{2} x_{1}=f \mathrm{e}^{\frac{1}{2} \mathrm{i} r \dot{\Omega} t^{2}} \mathrm{e}^{-\mathrm{i} \frac{\alpha}{2}} \\
& \ddot{x}_{2}+2 \xi(1+\Delta) \bar{\omega} \dot{x}_{2}+(1+\Delta)^{2} \bar{\omega}^{2} x_{2}=f \mathrm{i}^{\frac{1}{2} \mathrm{i} r \dot{\Omega} t^{2}} \mathrm{e}^{-\mathrm{i} \frac{\alpha}{2}}
\end{aligned}
$$

where

$$
\bar{\omega}=\frac{\omega_{r, 1}+\omega_{r, 2}}{2} ; \quad \Delta=\frac{\omega_{r, 2}-\omega_{r, 1}}{\omega_{r, 1}+\omega_{r, 2}}
$$

The relationship between the SW amplitudes and the TW amplitudes can be obtained by substituting Eqs. (12) into Eqs. (7). It yields:

$$
y_{1}=\frac{x_{1}-\mathrm{i} x_{2}}{2} \mathrm{e}^{\mathrm{i} \frac{\alpha}{2}} ; \quad y_{2}=\frac{x_{1}+\mathrm{i} x_{2}}{2} \mathrm{e}^{-\mathrm{i} \frac{\alpha}{2}}
$$

The manipulation of Eqs. (13) according to Eqs. (15) provides the equations of motion in terms of TW coordinates as:

$$
\left[\begin{array}{l}
\ddot{y}_{1} \\
\ddot{y}_{2}
\end{array}\right]+2 \xi \bar{\omega}\left[\begin{array}{cc}
1 & -\Delta \mathrm{e}^{\mathrm{i} \alpha} \\
-\Delta \mathrm{e}^{-\mathrm{i} \alpha} & 1
\end{array}\right]\left[\begin{array}{l}
\dot{y}_{1} \\
\dot{y}_{2}
\end{array}\right]+\bar{\omega}^{2}\left[\begin{array}{cc}
1+\Delta^{2} & -2 \Delta \mathrm{e}^{\mathrm{i} \alpha} \\
-2 \Delta \mathrm{e}^{\mathrm{i} \alpha} & 1+\Delta^{2}
\end{array}\right]\left[\begin{array}{l}
y_{1} \\
y_{2}
\end{array}\right]=\left[\begin{array}{c}
f \mathrm{e}^{\frac{1}{2} \mathrm{i} r \dot{\partial} t^{2}} \\
0
\end{array}\right]
$$

It can be observed that, while the equations in term of SW coordinates remain decoupled, the equations in terms of TW coordinates become coupled due to terms that scale with the mistuning amplitude.

For a systematic analysis of the problem, Eqs. (13) and (16) are non-dimensionalized introducing the scaling 


$$
\begin{array}{lll}
t \leftarrow \bar{\omega} t ; & \Omega \leftarrow \frac{\Omega}{\bar{\omega}} ; & \dot{\Omega} \leftarrow \frac{\dot{\Omega}}{\bar{\omega}^{2}} \\
y_{j} \leftarrow \mathrm{i} \frac{2 \xi}{f} y_{j} ; & x_{j} \leftarrow \mathrm{i} \frac{2 \xi}{f} x_{j} &
\end{array}
$$

Besides, the harmonic index $r$ is set equal to 1 without introducing any conceptual restriction. After these modifications, the equations of motion of become:

$$
\begin{aligned}
& {\left[\begin{array}{l}
\ddot{x}_{1} \\
\ddot{x}_{2}
\end{array}\right]+2 \xi\left[\begin{array}{cc}
1-\Delta & 0 \\
0 & 1+\Delta
\end{array}\right]\left[\begin{array}{l}
\dot{x}_{1} \\
\dot{x}_{2}
\end{array}\right]+\left[\begin{array}{cc}
(1-\Delta)^{2} & 0 \\
0 & (1+\Delta)^{2}
\end{array}\right]\left[\begin{array}{l}
x_{1} \\
x_{2}
\end{array}\right]=2 \xi \mathrm{i}\left[\begin{array}{c}
\mathrm{e}^{\frac{1}{2} \mathrm{i} \dot{\Omega} t^{2}} \mathrm{e}^{-\mathrm{i} \frac{\alpha}{2}} \\
\mathrm{i} \mathrm{e}^{\frac{1}{2} \mathrm{i} \dot{\Omega} t^{2}} \mathrm{e}^{-\mathrm{i} \frac{\alpha}{2}}
\end{array}\right]} \\
& {\left[\begin{array}{c}
\ddot{y}_{1} \\
\ddot{y}_{2}
\end{array}\right]+2 \xi\left[\begin{array}{cc}
1 & -\Delta \mathrm{e}^{\mathrm{i} \alpha} \\
-\Delta \mathrm{e}^{-\mathrm{i} \alpha} & 1
\end{array}\right]\left[\begin{array}{l}
\dot{y}_{1} \\
\dot{y}_{2}
\end{array}\right]+\left[\begin{array}{cc}
1+\Delta^{2} & -2 \Delta \mathrm{e}^{\mathrm{i} \alpha} \\
-2 \Delta \mathrm{e}^{\mathrm{i} \alpha} & 1+\Delta^{2}
\end{array}\right]\left[\begin{array}{l}
y_{1} \\
y_{2}
\end{array}\right]=2 \xi \mathrm{i}\left[\begin{array}{c}
\mathrm{e}^{\frac{1}{2} \mathrm{i} \dot{\Omega} t^{2}} \\
0
\end{array}\right]}
\end{aligned}
$$

The scaled model depends on the damping ratio $\xi$, the mistuning ratio $\Delta$, the (non-dimensional) chirp rate $\dot{\Omega}$ and the mode clocking angle $\alpha$.

\section{QUASI-STEADY RESPONSE OF A TUNED SYSTEM}

If the system is tuned (i.e. $\Delta=0$ ) and if the chirp rate $\dot{\Omega}$ is very small (to be quantified next), the system response can be obtained as the steady-state response due to a harmonic load of frequency $\Omega=\dot{\Omega} t$, i.e.:

$$
\left[\begin{array}{l}
\ddot{y}_{1} \\
\ddot{y}_{2}
\end{array}\right]+2 \xi\left[\begin{array}{l}
\dot{y}_{1} \\
\dot{y}_{2}
\end{array}\right]+\left[\begin{array}{l}
y_{1} \\
y_{2}
\end{array}\right]=2 \xi \mathrm{i}\left[\begin{array}{c}
\mathrm{e}^{\mathrm{i} \Omega t} \\
0
\end{array}\right]
$$

where only $y_{1}$ shows a resonance at $\Omega=1$, while $y_{2}$ remains $\sim \mathcal{O}(\xi)$ and can be neglected. The solution is readily available in the form:

$$
y_{1}=A \mathrm{e}^{\mathrm{i} \Omega t} ; \quad A=\frac{2 \xi \mathrm{i}}{1-\Omega^{2}+2 \mathrm{i} \xi \Omega}
$$


showing that, for $\xi \ll 1$, the maximum amplitude appears at $t=1 / \dot{\Omega}$ and is equal to 1

$$
A_{Q S-\text { tuned }}=\max |A|=1
$$

Figure 1 shows the response of a bladed disk with $N=6$ blades and $\xi=3 \cdot 10^{-3}$. The amplitude $|A|$ is reported together with the displacement $u_{j}$ of the individual blades. It can be observed that all the blades share the same envelope, but vibrate with a different phase angle.

\section{TRANSIENT RESPONSE OF A TUNED SYSTEM}

If the sweep velocity $\dot{\Omega}$ is not very small, transient effects during the resonance crossing appear. This situation is typical of the resonance crossing of rotors during run-up or run-down. The effects are (1) a shift of the amplification peak towards high frequencies for run-up and low frequencies for rundown; (2) reduction of the peak height; (3) increment of the peak width; (4) amplitude modulation after the resonance crossing due to the beating of the transient free-decaying response and the forced response (e.g. [1-5]).

The equation of motion is obtained from Eq. (19) by letting $\Delta=0$ and reads

$$
\left[\begin{array}{c}
\ddot{y}_{1} \\
\ddot{y}_{2}
\end{array}\right]+2 \xi\left[\begin{array}{c}
\dot{y}_{1} \\
\dot{y}_{2}
\end{array}\right]+\left[\begin{array}{c}
y_{1} \\
y_{2}
\end{array}\right]=2 \xi \mathrm{i}\left[\begin{array}{c}
\mathrm{e}^{\mathrm{i} \frac{1}{2} \dot{\Omega} t^{2}} \\
0
\end{array}\right]
$$

Likewise for Eq. (20), $y_{2}$ is not excited by the load and can be disregarded. Besides, it is convenient to first shift the time to the vicinity of the resonance as $t \leftarrow t-t_{0}$, with $t_{0}=1 / \dot{\Omega}$. With this substitution, the equation of motion becomes:

$$
\ddot{y}_{1}+2 \xi \dot{y}_{1}+y_{1}=2 \xi \mathrm{ie} \mathrm{e}^{\mathrm{i} \frac{1}{2 \dot{\Omega}}} \mathrm{e}^{\mathrm{i} t} \mathrm{e}^{\mathrm{i} \frac{1}{2} \dot{\Omega} t^{2}}
$$


The first exponential term in the forcing gives a constant phase change on the solution, and can be removed by introducing the variable

$$
y_{1}=Y \mathrm{e}^{\frac{\mathrm{i}}{2 \dot{\Omega}}}
$$

After this substitution, the equation of motion becomes:

$$
\ddot{Y}+2 \xi \dot{Y}+Y=2 \xi \mathrm{ie} \mathrm{e}^{\mathrm{i} t} \mathrm{e}^{\mathrm{i} \frac{1}{2} \dot{\Omega} t^{2}}
$$

The small damping $\xi \ll 1$ and chirp rate $\dot{\Omega} \ll 1$ produce a slow time modulation of the solution that can be captured using the multiple scales method (see, e.g. [16-17]). To this purpose, it is necessary to formalize the relative smallness of the two parameters by letting, without loss of generality, $\xi=\varepsilon$ and $\dot{\Omega}=\kappa \varepsilon^{q}$ where $0<\varepsilon \ll 1, \kappa \sim 1$ and $q>0$.

$$
\ddot{Y}+2 \varepsilon \dot{Y}+Y=2 \varepsilon \mathrm{ie}^{\mathrm{i} t} \mathrm{e}^{\mathrm{i} \frac{1}{2} \kappa \varepsilon^{q} t^{2}}
$$

The solution is expanded using two time scales $t \sim 1$ and $\tau=\varepsilon t$

$$
Y=Y^{0}(t, \tau)+\varepsilon Y^{1}(t, \tau)+\ldots
$$

and, inserted into Eq. (27), equating terms with the same power of $\varepsilon$, gives:

$$
\begin{array}{ll}
\operatorname{ord}\left(\varepsilon^{0}\right): & \partial_{t}^{2} Y^{0}+Y^{0}=0 \\
\operatorname{ord}\left(\varepsilon^{1}\right): & \partial_{t}^{2} Y^{1}+Y^{1}+2 \partial_{t \tau} Y^{0}+2 \partial_{t} Y^{0}=2 \mathrm{ie}^{\mathrm{i} t} \mathrm{e}^{\mathrm{i} \frac{1}{2} \kappa \varepsilon^{q} t^{2}}
\end{array}
$$

The solution at the leading order is directly available and reads

$$
Y^{0}=A(\tau) \mathrm{e}^{\mathrm{i} t}
$$


where the amplitude $A(\tau)$ is a function of the slow time scale and will be determined with a secularity condition. As far as the order $\varepsilon^{1}$ is concerned, it must be noted that the governing equation should not depend explicitly on the small parameter $\varepsilon$. It is therefore necessary to re-write the right-hand side of Eq. (29) by choosing $\varepsilon^{q} t^{2}=t^{\beta} \tau^{\gamma}$ so that the parameter $\varepsilon$ disappears. Substituting $\tau=\varepsilon t$ indicates that it is necessary to choose $\gamma=q$ and $\beta+\gamma=2$, so that the governing equation at the order $\varepsilon$ becomes

$$
\partial_{t}^{2} Y^{1}+Y^{1}=-2 \mathrm{ie}^{\mathrm{i} t}\left(\frac{d A}{d \tau}+A\right)+2 \mathrm{ie}^{\mathrm{i} t \mathrm{e}^{\mathrm{i} \frac{1}{2} \kappa t^{2-q} \tau^{q}}}
$$

To prevent the presence of secular terms, to assure that $Y_{1}$ is finite, it is necessary to force to zero the resonant terms present at the RHS. If $q \neq 2$ this condition leads to the equation

$$
\frac{d A}{d \tau}+A=0
$$

which, with the initial condition $A \rightarrow 0$ at $\tau \rightarrow-\infty$, only admits the trivial solution $A=0$. In order to find a non-trivial solution, it must be set $q=2$, thus the secularity condition reads:

$$
\frac{d A}{d \tau}+A=\mathrm{e}^{\mathrm{i} \frac{1}{2} \kappa \tau^{2}}
$$

The general solution of this linear problem can be written as

$$
A(\tau)=C \mathrm{e}^{-\tau}+\int_{-\infty}^{\tau} \mathrm{e}^{\mathrm{i} \frac{1}{2} \kappa s^{2}-(\tau-s)} d s
$$

where the constant $C$ can be set to zero to select the solution that verifies $A \rightarrow 0$ as $\tau \rightarrow-\infty$. Moreover, the Faddeeva function [18] 


$$
w(z)=\mathrm{e}^{-z^{2}}\left(1+\frac{2 \mathrm{i}}{\sqrt{\pi}} \int_{0}^{z} \mathrm{e}^{\zeta^{2}} d \zeta\right)
$$

allows to finally express the envelope $A(\tau)$ in the form

$$
A(\tau)=-\sqrt{\frac{\pi \mathrm{i}}{2 \kappa}} \mathrm{e}^{\mathrm{i} \frac{1}{2} \kappa \tau^{2}} w\left(\tau \sqrt{\frac{\mathrm{i} \kappa}{2}}+\frac{1}{\sqrt{2 \mathrm{i} \kappa}}\right) \quad\left(\text { with } \sqrt{\mathrm{i}}=-\frac{1+\mathrm{i}}{\sqrt{2}}\right)
$$

which gives a much more straightforward way to compute the response. Substituting Eq. (36) back into Eqs. (30), (28), (25) and (10), the response can be expressed as

$$
\left[\begin{array}{l}
y_{1} \\
y_{2}
\end{array}\right]=\left[\begin{array}{l}
1 \\
0
\end{array}\right] A(\tau) \mathrm{e}^{\frac{\mathrm{i}}{2 \dot{\Omega}}} \mathrm{e}^{\mathrm{i} t} ; \quad\left[\begin{array}{l}
x_{1} \\
x_{2}
\end{array}\right]=\left[\begin{array}{l}
1 \\
\mathrm{i}
\end{array}\right] A(\tau) \mathrm{e}^{\frac{\mathrm{i}}{2 \dot{\Omega}}} \mathrm{e}^{\mathrm{i} t}
$$

This result provides a method to calculate the transient response of the system, but also indicates that the right scaling for the chirp rate is obtained by choosing $\dot{\Omega}=\kappa \varepsilon^{2}$. This suggests that $\kappa=\dot{\Omega} / \xi^{2}$ is the correct parameter that should be employed to represent the chirp rate, as it was already deduced in [4] on the basis of different arguments.

Figure 2 shows the transient response of a tuned disk with $N=6, \xi=3 \cdot 10^{-3}$ and $\kappa=10$. The blade response $u_{j}$ and the TW coordinate $y_{1}$ are obtained by numerical integration of Eqs. (23) and (7), while the envelope $A$ is calculated by the asymptotic solution (36). To save computational time, the numerical integration is started with zero initial conditions at the time $t_{\text {start }}=0.8 t_{0}$. It was verified that this choice makes the effects of the initial conditions at the resonance crossing negligible for the whole considered parameter space.

It can be observed that, likewise in the QS case, the vibration of all the blades share the same envelope, which is very well approximated by the asymptotic solution, even for the considered case with relatively high chirp rate. It can be appreciated that the maximum response amplitude is below the 
maximum QS response $A_{Q S-t u n e d}$. This fact is general and can be proved analytically by multiplying Eq. (33) by $A^{*}$ and adding the complex conjugate of the equation to obtain the energy equation

$$
2 \frac{d|A|^{2}}{d \tau}+2|A|^{2}=A^{*} \mathrm{e}^{\mathrm{i} \frac{1}{2} \kappa \tau^{2}}+A \mathrm{e}^{-\mathrm{i} \frac{1}{2} \kappa \tau^{2}}
$$

As the maximum amplitude appears for $\mathrm{d}|A| / \mathrm{d} \tau=0$, therefore:

$$
2|A|^{2}=A^{*} \mathrm{e}^{\mathrm{i} \frac{1}{2} \kappa \tau^{2}}+A \mathrm{e}^{-\mathrm{i} \frac{1}{2} \kappa \tau^{2}}
$$

Then, using the complex variable $B=A \mathrm{e}^{-\mathrm{i} \kappa \tau^{2} / 2}$ and its real and imaginary parts $B=R+\mathrm{i} I$, Eq. (39) simplifies to

$$
R^{2}+I^{2}=R
$$

which corresponds to a circumference in the complex plane with maximum distance to the origin equal to 1 . This prove that:

$$
|A|=|B|=\sqrt{R^{2}+I^{2}} \leq 1
$$

Figure 3 shows the maximum response $u_{j}$ as a function of $\kappa$, calculated by numerical integration of Eq. (24) for different values of the damping $\xi$ ranging between $10^{-3}, 10^{-2}$, compared with the maximum amplitude provided by the asymptotic solution. The perfect matching of the results confirms that the asymptotic solution is accurate in a very wide range of $\kappa$ and that the system response does not depend on the $\dot{\Omega}$ and $\xi$ separately, but only jointly through $\kappa$. Besides, Figure 3 demonstrates that transient effects vanish when $\kappa \ll 1$. 


\section{QUASI-STEADY RESPONSE OF A MISTUNED SYSTEM}

When mistuning is present, the equations of motion in terms of TW coordinates become coupled and also $y_{2}$, which is not directly forced, plays a role in the response. If the chirp rate is very small, the response of Eq. (19) approaches its steady-state and can be written in the form

$$
\left[\begin{array}{l}
y_{1} \\
y_{2}
\end{array}\right]=\left[\begin{array}{l}
A_{1} \\
A_{2}
\end{array}\right] \mathrm{e}^{\mathrm{i} \Omega t}
$$

where $\Omega=\dot{\Omega} t$, and the amplitudes $A_{1}$ and $A_{2}$ can be obtained by manipulating the system FRF. For $\xi, \Delta \ll 1$, it yields

$$
\begin{aligned}
& A_{1}=\frac{-2 \xi \mathrm{i}\left(-\Omega^{2}+2 \mathrm{i} \xi \Omega+1\right)}{-\Omega^{4}+4 \mathrm{i} \xi \Omega^{3}+2\left(1+\Delta^{2}+2 \xi^{2}\right) \Omega^{2}-4 \mathrm{i} \xi \Omega+2 \Delta^{2}-1} \\
& A_{2}=\frac{-4 \Delta \xi \mathrm{i} \mathrm{e}^{-\mathrm{i} \alpha}}{-\Omega^{4}+4 \mathrm{i} \xi \Omega^{3}+2\left(1+\Delta^{2}+2 \xi^{2}\right) \Omega^{2}-4 \mathrm{i} \xi \Omega+2 \Delta^{2}-1}
\end{aligned}
$$

Due to the definition of the TW coordinates, the quantity $\left|A_{1}+A_{2}\right|$ represents the vibration amplitude of the blade 0 and depends on the angle of the mistuning pattern $\alpha$. Besides, the angle $\alpha$ also controls the relative phase angle between $A_{1}$ and $A_{2}$, in such a way that it is always possible to select a value of $\alpha$ for which $A_{1}$ and $A_{2}$ are aligned in the complex plane. This condition provides the maximum of $\left|A_{1}+A_{2}\right|$ and the envelope of the blade vibration

$$
A_{Q S}=\max _{\alpha}\left|A_{1}+A_{2}\right|=\left|A_{1}\right|+\left|A_{2}\right| \cdot
$$

Figure 4 shows the QS response of a disk with $N=27$ blades, $\xi=3 \cdot 10^{-3}, \Delta=4.4 \cdot 10^{-3}$. The detail of the figure shows that the envelope is reached only by a few blades, while the others vibrates with a lower amplitude. $A_{Q S}$ depends on $\Omega$, which in turns depends on $t$. The maximum amplitude of vibration 
occurring during the resonance crossing can be obtained by maximizing numerically Eqs. (43)-(44) with respect to $\Omega$.

Figure 5 shows the maximum amplitude of vibration as a function of $\xi$ and $\Delta$. It can be noted that for $\Delta / \xi \rightarrow 0$ the maximum response tends to 1 , the QS response of the tuned system. The tip of the curve is consistent with the Whitehead's limit $(1+\sqrt{2}) / 2$. For small levels of mistuning the maximum response is determined only by the ratio $\Delta / \xi$, while when $\Delta \sim \xi$ their separate influence is visible, though relatively small.

\section{TRANSIENT RESPONSE OF A MISTUNED SYSTEM}

Eq. (19) contains the small parameter $\Delta$, beside the two parameters $\xi$ and $\dot{\Omega}$ already discussed for the tuned case. Like in the previous section, we formalize the relative smallness of this new parameter by setting $\Delta=m \mathrm{ie}^{-\mathrm{i} \alpha} \varepsilon^{p}$ where $|m| \sim 1$ and $p>0$ is to be determined in order to obtain a distinguished limit. Besides, the chirp rate is set to $\dot{\Omega}=\kappa \varepsilon^{2}$ according to the findings of the previous section, the time is shifted to the resonance condition $t \leftarrow t-t_{0}$, and the resulting constant phase is absorbed in the new variables

$$
y_{1}=Y_{1} \mathrm{e}^{\frac{\mathrm{i}}{2 \dot{\Omega}}} ; \quad y_{2}=Y_{2} \mathrm{e}^{\frac{\mathrm{i}}{2 \dot{\Omega}}}
$$

After this substitution the equations of motion result

$$
\left[\begin{array}{c}
\ddot{Y}_{1} \\
\ddot{Y}_{2}
\end{array}\right]+2 \varepsilon\left[\begin{array}{cc}
1 & -\mathrm{i} \varepsilon^{p} m \\
\mathrm{i} \varepsilon^{p} m^{*} & 1
\end{array}\right]\left[\begin{array}{l}
\dot{Y}_{1} \\
\dot{Y}_{2}
\end{array}\right]+\left[\begin{array}{cc}
1+\varepsilon^{2 p}|m|^{2} & -2 \mathrm{i} \varepsilon m \\
2 \mathrm{i} \varepsilon m^{*} & 1+\varepsilon^{2 p}|m|^{2}
\end{array}\right]\left[\begin{array}{l}
Y_{1} \\
Y_{2}
\end{array}\right]=2 \varepsilon \mathrm{i}\left[\begin{array}{c}
\mathrm{e}^{\mathrm{i} t} \mathrm{e}^{\mathrm{i} \frac{1}{2} \kappa \tau^{2}} \\
0
\end{array}\right]
$$

The solution is then expanded using the two time scales $t \sim 1$ and $\tau=\varepsilon t$

$$
\left[\begin{array}{l}
Y_{1} \\
Y_{2}
\end{array}\right]=\left[\begin{array}{l}
Y_{1}^{0}(t, \tau) \\
Y_{2}^{0}(t, \tau)
\end{array}\right]+\varepsilon\left[\begin{array}{l}
Y_{1}^{1}(t, \tau) \\
Y_{2}^{1}(t, \tau)
\end{array}\right]+\ldots
$$


and inserted into Eq. (46) to obtain, equating the terms at the same power of $\varepsilon$ :

$$
\begin{aligned}
& \operatorname{ord}\left(\varepsilon^{0}\right):\left[\begin{array}{l}
\partial_{t}^{2} Y_{1}^{0} \\
\partial_{t}^{2} Y_{2}^{0}
\end{array}\right]+\left[\begin{array}{l}
Y_{1}^{0} \\
Y_{2}^{0}
\end{array}\right]=\left[\begin{array}{l}
0 \\
0
\end{array}\right] \\
& \operatorname{ord}\left(\varepsilon^{p}\right):\left[\begin{array}{cc}
0 & -2 \mathrm{i} m \\
2 \mathrm{i} m^{*} & 0
\end{array}\right]\left[\begin{array}{l}
Y_{1}^{0} \\
Y_{2}^{0}
\end{array}\right]=\left[\begin{array}{l}
0 \\
0
\end{array}\right] \\
& \operatorname{ord}\left(\varepsilon^{1}\right):\left[\begin{array}{l}
\partial_{t}^{2} Y_{1}^{1} \\
\partial_{t}^{2} Y_{2}^{1}
\end{array}\right]+\left[\begin{array}{l}
Y_{1}^{1} \\
Y_{2}^{1}
\end{array}\right]+2\left[\begin{array}{l}
\partial_{t \tau} Y_{1}^{0} \\
\partial_{t \tau} Y_{2}^{0}
\end{array}\right]+2\left[\begin{array}{l}
\partial_{t} Y_{1}^{0} \\
\partial_{t} Y_{2}^{0}
\end{array}\right]=2 \mathrm{i}\left[\begin{array}{c}
\mathrm{e}^{\mathrm{i} t} \mathrm{e}^{\mathrm{i} \frac{1}{2} \kappa \tau^{2}} \\
0
\end{array}\right]
\end{aligned}
$$

with the following order being $\varepsilon^{1+p}$. Since $p>0$, the governing equation at the leading order is the first of Eqs. (48), whose solution can be expressed as

$$
\left[\begin{array}{l}
Y_{1}^{0} \\
Y_{2}^{0}
\end{array}\right]=\left[\begin{array}{l}
A_{1}(\tau) \\
A_{2}(\tau)
\end{array}\right] \mathrm{e}^{\mathrm{i} t}
$$

The second of Eqs. (48), at the order $\varepsilon^{p}$, cannot be fulfilled unless by the trivial solution. For this reason, $p$, which is still to be defined, cannot be lower than 1 . On the other hand, if it were $p>1$, then the third of Eqs. (48) would be the next-order equation and the solution would result independent of the mistuning. Consequently, the condition to find a non-trivial solution depending on mistuning is setting $p=1$. With this condition, the second and the third of Eqs. (48) combine and the next-order problem reads

$$
\left[\begin{array}{l}
\partial_{t}^{2} Y_{1}^{1} \\
\partial_{t}^{2} Y_{2}^{1}
\end{array}\right]+\left[\begin{array}{l}
Y_{1}^{1} \\
Y_{2}^{1}
\end{array}\right]=2 \mathrm{i} \mathrm{e}^{\mathrm{i} t}\left(\left[\begin{array}{c}
\mathrm{i} \frac{1}{2} \kappa \tau^{2} \\
0
\end{array}\right]-\left[\begin{array}{c}
\partial_{\tau} A_{1} \\
\partial_{\tau} A_{2}
\end{array}\right]-\left[\begin{array}{cc}
1 & -m \\
m^{*} & 1
\end{array}\right]\left[\begin{array}{l}
A_{1} \\
A_{2}
\end{array}\right]\right)
$$

The cancellation of the terms proportional to $\mathrm{e}^{\mathrm{i} t}$ to impose the secularity condition gives the following system of equations for the slow time evolution of the amplitudes $A_{1}$ and $A_{2}$ 


$$
\left[\begin{array}{l}
\partial_{\tau} A_{1} \\
\partial_{\tau} A_{2}
\end{array}\right]+\left[\begin{array}{cc}
1 & -m \\
m^{*} & 1
\end{array}\right]\left[\begin{array}{l}
A_{1} \\
A_{2}
\end{array}\right]=\left[\begin{array}{c}
\mathrm{i} \frac{1}{2} \kappa \tau^{2} \\
0
\end{array}\right]
$$

The solution of this linear system that verifies $A_{1}, A_{2} \rightarrow 0$ at $\tau \rightarrow-\infty$ can be expressed in closed form using again the Faddeeva function as

$$
\left[\begin{array}{l}
A_{1} \\
A_{2}
\end{array}\right]=\frac{1}{2}\left[\begin{array}{cc}
1 & 1 \\
\mathrm{e}^{-\mathrm{i} \alpha} & -\mathrm{e}^{-\mathrm{i} \alpha}
\end{array}\right]\left[\begin{array}{l}
I_{1} \\
I_{2}
\end{array}\right]
$$

where

$$
I_{1,2}=-\sqrt{\frac{\pi \mathrm{i}}{2 \kappa}} \mathrm{e}^{\mathrm{i} \frac{1}{2} \kappa \tau^{2}} w\left(\tau \sqrt{\frac{\mathrm{i} \kappa}{2}}+(1 \pm \mathrm{i}|m|) \frac{1}{\sqrt{2 \mathrm{i} \kappa}}\right)
$$

The dynamic response can be obtained by substituting Eqs. (52) and (53) back into Eqs. (49), (47), (45) and (15).

$$
\begin{gathered}
{\left[\begin{array}{l}
y_{1} \\
y_{2}
\end{array}\right]=\frac{1}{2}\left[\begin{array}{c}
I_{1}+I_{2} \\
\left(I_{1}-I_{2}\right) \mathrm{e}^{-\mathrm{i} \alpha}
\end{array}\right] \mathrm{e}^{\frac{\mathrm{i}}{2 \dot{\Omega}}} \mathrm{e}^{\mathrm{i} t}} \\
{\left[\begin{array}{l}
x_{1} \\
x_{2}
\end{array}\right]=\frac{1}{2}\left[\begin{array}{c}
I_{1} \\
\mathrm{i} I_{2}
\end{array}\right] \mathrm{e}^{\frac{\mathrm{i}}{2 \dot{\Omega}}} \mathrm{e}^{-\mathrm{i} \frac{\alpha}{2}} \mathrm{e}^{\mathrm{i} t}}
\end{gathered}
$$

The displacement of the blade $j=0$ is given by the quantity $\left|A_{1}+A_{2}\right|$, thus the computation of the maximum response requires to maximize the quantity

$$
\left|A_{1}+A_{2}\right|=\frac{1}{2}\left|\left(I_{1}+I_{2}\right)+\mathrm{ie}^{-\mathrm{i} \alpha}\left(I_{1}-I_{2}\right)\right|
$$

over $\tau, \kappa,|m|, \alpha$. The maximization over $\alpha$ can be easily performed as 


$$
A=\max _{\alpha}\left|A_{1}+A_{2}\right|=\frac{1}{2}\left|I_{1}+I_{2}\right|+\frac{1}{2}\left|I_{1}-I_{2}\right|
$$

because the modulus of the sum of the two complex numbers $\left(I_{1}+I_{2}\right)$ and $\left(I_{1}-I_{2}\right)$ will reach its maximum when they are aligned.

Figure 6 shows the transient response of a mistuned disk, as well as its envelope calculated through the TW coordinates and the asymptotic solution. The parameters employed for the calculation are $N=27, \xi=3 \cdot 10^{-3},|m|=1.5$ and $\kappa=10$. The blade displacement $u_{j}$ and the TW coordinates are calculated by numerical integration, while the envelope $A$ is obtained from Eq. (57). It can be observed that the asymptotic solution is very accurate.

$A$ represents the maximum amplitude of vibration in the disk (i.e. the amplitude of worst blade) at a given time $\tau$, for a specified chirp rate $\kappa$ and mistuning intensity $|m|$. The maximum response during the whole resonance crossing is obtained by maximizing $A$ with respect to $\tau$. Figure 7 shows this result (solid lines), compared by the maximum response calculated by numerical integration of Eqs. (19) and expressed as $\max \left(\left|y_{1}\right|+\left|y_{2}\right|\right)$ (dashed lines). These latter results are provided for 8 values of damping in the range $\left[10^{-3}, 10^{-2}\right]$. The maximum amplification of the transient response because of mistuning is always below the maximum mistuning amplification for the QS case (Whitehead's limit), but, on the other hand, for a given chirp rate, $\kappa=$ fixed, mistuning amplification with respect to the tuned transient response can be much larger than Withehead's limit.

It has been observed that chirp rate and mistuning play an opposite role on the response amplification in resonance crossing. Namely, while the transient effects due to a non-zero chirp rate lead to a reduction of the response, mistuning produces an increment of the amplitude. However, these two phenomena are not decoupled as the ability of mistuning in over-amplifying the resonant response increases as the chirp rate increases. This circumstance can be observed in Figure 8 representing the ratio between the maximum transient response of a mistuned system (function of $|m|$ and $\kappa$ ) and the maximum transient response of a tuned system driven with the same chirp rate. It can be observed 
that the amplification due to mistuning is well above the Whitehead's limit in a very wide region of the parameter space; and it grows as $|m|$ and $\kappa$ become larger.

The following two ideas could give an explanation for this (a priori surprising) mistuning amplification larger than in the QS case:

1. As mistuning is increased, the two mistuned resonant frequencies split. For a fixed amount of mistuning (vertical line in Figure 8), if the frequency sweep is too slow, the first excited mistuned mode has decayed too much when the sweep arrives to the second resonance and there is no possible interaction between the two modes to generate amplification. Only if the sweep is appropriately fast then there could be interaction between the two mistuned modes that can lead to an amplification of the response.

2. On the other hand, the tuned amplitude (which is in the denominator of the amplification ratio) is reduced as the frequency ramp becomes faster. For high chirp rates the maximum response can be much smaller than 1 (see Figure 3), but, in the QS case, the maximum tuned response is fixed to 1 (see Figure 1).

Figure 9 shows the ratio of the transient response of the mistuned system and its QS response. It can be observed that, in a relatively narrow region of the parameter space the ratio is above unity indicating that, for the same system, the transient response is larger than the QS response.

\section{CONCLUSIONS}

When the vibration of a bladed disk is governed by a pair of isolated modes, the system can be represented as a 2-dof linear oscillator. For this system, we derived asymptotic solutions valid both for the tuned and the mistuned case. This result has consequences both for the quantitative and qualitative analysis of bladed disks in resonance crossing condition.

From a quantitative point of view, the asymptotic solutions enable a fast calculation of the transient response avoiding the numerical integration of the equations of motion. 
From a qualitative point of view, the development demonstrates that the problem is governed by two non-dimensional parameters, namely, the reduced chirp rate $\kappa=\dot{\Omega} / \xi^{2}$ and the mistuning parameter $m=\Delta / \xi$. These two parameters provide a proper quantification of the importance of transient effects and mistuning, enabling the judgment and the comparison of systems under this point of view.

The asymptotic solutions, as well as the numerical simulation showed that the amplification due to mistuning is significantly more effective in transient conditions than in QS condition. This circumstance has two consequences. First, the increment of amplitude due to mistuning should be evaluated using a transient formulation as the use of the QS increment is not on the safe side. Second, the design of mistuned disks in resonance crossing should not rely on the beneficial effects of a fast resonance crossing. In the most unfavorable case, the transient effects can even over-amplify the QS response producing an effect that is opposite to the usual one.

\section{ACKNOWLEDGMENTS}

The work of Carlos Martel has been supported by the Spanish Ministerio de Ciencia, Innovación y Universidades under grant DPI2017-84700-R.

\section{REFERENCES}

[1] Lewis, F. M., 1932, "Vibration during acceleration through a critical speed," ASME J. Appl. Mech., 54, pp. 258-261.

[2] Markert, R., and Seidler, M., 2001, “Analytically based estimation of the maximum amplitude during passage through resonance”, Int. J. Solids Struct., 38(10-13), pp. 1975-1992.

[3] Ayers, J.P., Feiner, D.M., and Griffin, J.H., 2006. “A Reduced-Order Model for Transient Analysis of Bladed Disk Forced Response", Journal of Turbomachinery, 128(3), pp. 466-473.

[4] Hackenberg, H., and Hartung, A., 2016. “An approach for estimating the effect of transient sweep through a resonance", J. Eng. Gas Turbines Power, 138(8), 082502. 
[5] Carassale, L., Marrè-Brunenghi, M., Patrone, S., 2018, "Wavelet-based identification of rotor blades in passage-through-resonance tests”, Mechanical Systems and Signal Processing, 98, pp. 124-138.

[6] Rivas-Guerra, A.J., and Mignolet, M.P., 2003. "Maximum amplification of blade response due to mistuning: localization and mode shape aspects of the worst disks", Journal of Turbomachinery, 125(3), pp. 442-454.

[7] Sinha, A., 2017. "Vibration of nearly periodic structures and mistuned bladed rotors". Cambridge University Press, Cambridge, UK.

[8] Whitehead, D., 1966, "effect of mistuning on the vibration of turbomachine blades induced by wakes", J. Mech. Eng. Sci., 8(1), pp. 15-21.

[9] Martel, C., and Corral, R., 2008. "Asymptotic description of maximum mistuning amplification of bladed disk forced response", J. Eng. Gas Turbines Power, 131(2), 022506.

[10]Kaneko, Y., 2013. "Study on transient vibration of mistuned bladed disk passing through resonance", Proceedings of ASME Turbo Expo 2013. Paper \# GT2013-94052.

[11]Bonhage, M., Pohle, L., Panning-von Scheidt, L., and Wallaschek, J., 2015. “Transient amplitude amplification of mistuned blisks”, J. Eng. Gas Turbines Power, 137(11): 112502.

[12] Siewert, C., and Stüer, H., 2016. "Transient forced response analysis of mistuned steam turbine blades during startup and coastdown", J. Eng. Gas Turbines Power, 139(1): 012501.

[13]Bonhage, M., Adler, J.T., Kolhoff, C., Hentschel, O., Schlesier, K.-D., Panning-von Scheidt, L. and Wallaschek, J., 2018. "Transient amplitude amplification of mistuned structures: An experimental validation", Journal of Sound and Vibration, 436, pp 236-252.

[14] Schlesier, K.-D., Panning-von Scheidt, L. and Wallaschek, J., 2018. "Investigations on transient amplitude amplification by applying intentional mistuning", Proceedings of ASME Turbo Expo 2018. Paper \# GT2018-75514.

[15] Willeke, S., Schwerdt, L., Panning-von Scheidt, L. and Wallaschek, J., 2018. "Intentional 
response reduction by harmonic mistuning of bladed disks with aerodynamic damping”. J. Eng. Gas Turbines Power, 140(12), 121010-121010-10.

[16]Bender, C.M. and Orszag, S.A., 1999. "Advanced mathematical methods for scientists and engineers: I: Asymptotic methods and perturbation theory”. Springer, New York, USA.

[17]Kevorkian J.K. and Cole J.D., 1996. "Multiple Scale and Singular Perturbation Methods", Springer-Verlag, New York, USA.

[18] Abramovitz M. and Stegun I.A., 1965. "Handbook of Mathematical Functions", Dover Publications, New York, USA. 


\section{FIGURE CAPTIONS}

Figure 1. Quasi-steady response of a tuned disk. $N=6, \xi=3 \cdot 10^{-3}$.

Figure 2. Transient response of a tuned system; $u_{j}$ and $y_{1}$ calculated by numerical integration; $A$ calculated by asymptotic expansion. $N=6, \xi=3 \cdot 10^{-3}, \kappa=10$.

Figure 3. Maximum amplitude of vibration as a function of $\kappa$ calculated by numerical integration (dots) with 8 values of $\xi$ in the range $\left[10^{-3}, 10^{-2}\right]$ and asymptotic solution (solid line).

Figure 4. Quasi-steady response of a mistuned disk. $N=27, \xi=3 \cdot 10^{-3}, \Delta=4.4 \cdot 10^{-3}$.

Figure 5. Maximum QS response of a mistuned disk.

Figure 6. Transient response of a mistuned disk. $N=27, \xi=3 \cdot 10^{-3}, \kappa=10,|m|=1.5$.

Figure 7. Maximum transient response; $y_{1}$ and $y_{2}$ are calculated by numerical integration for 8 values of $\xi \in\left[10^{-3}, 10^{-2}\right]$ (thin concour lines); $A$ is obtained by the asymptotic solution (thick contour lines). Figure 8. Transient amplification with respect to the tuned disk.

Figure 9. Transient amplification with respect to the QS response (TAMS). 
Figure 1

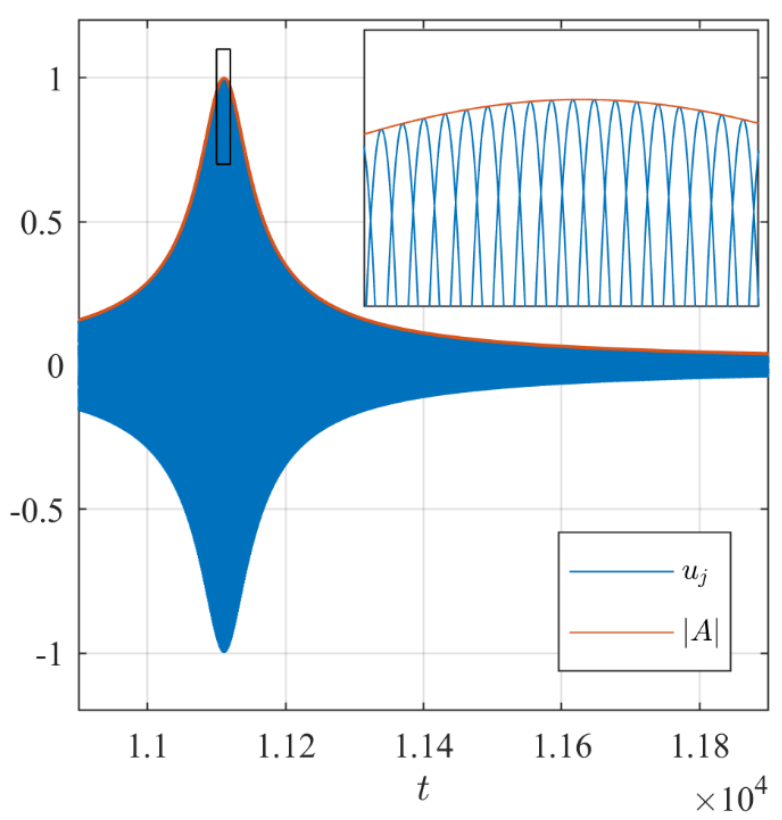


Figure 2

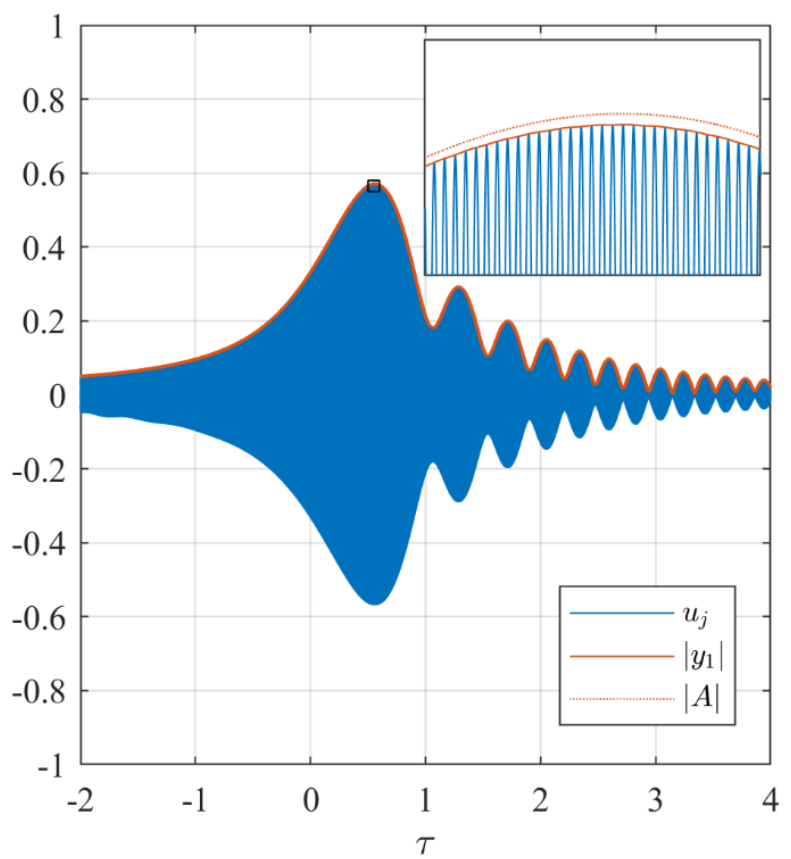


Figure 3

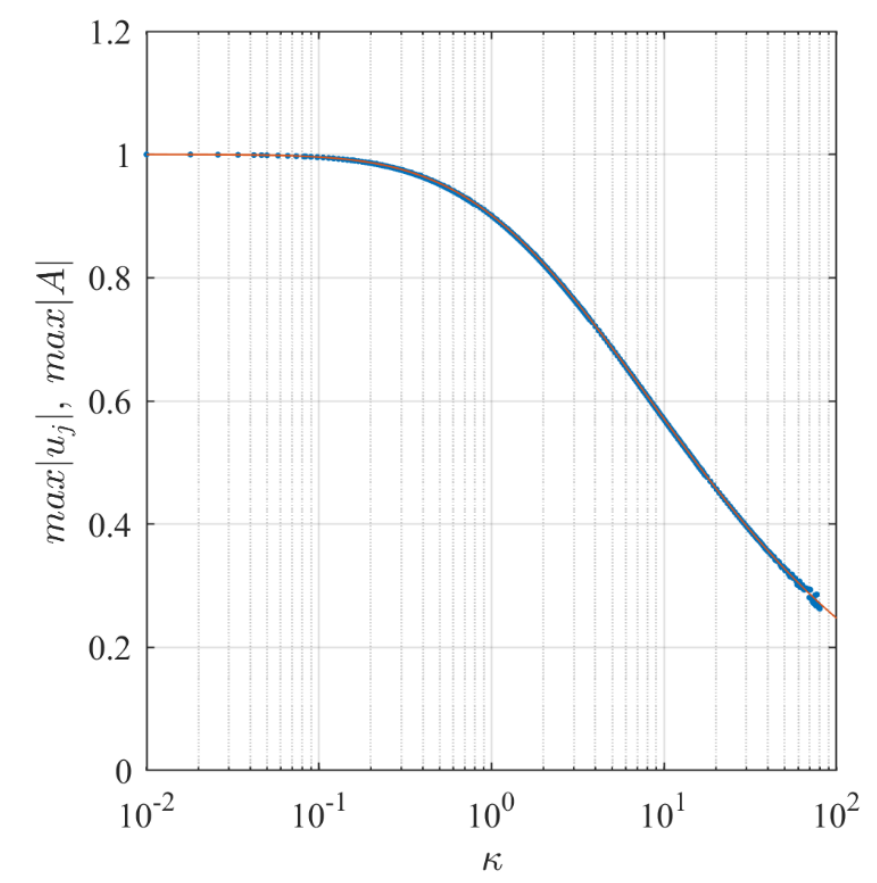


Figure 4

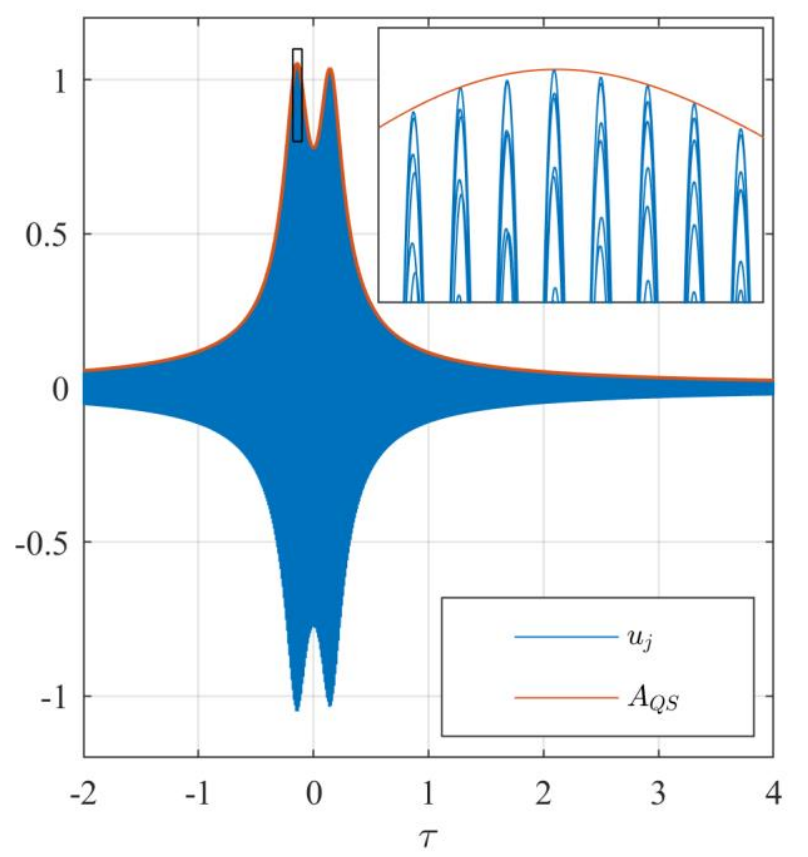


Figure 5

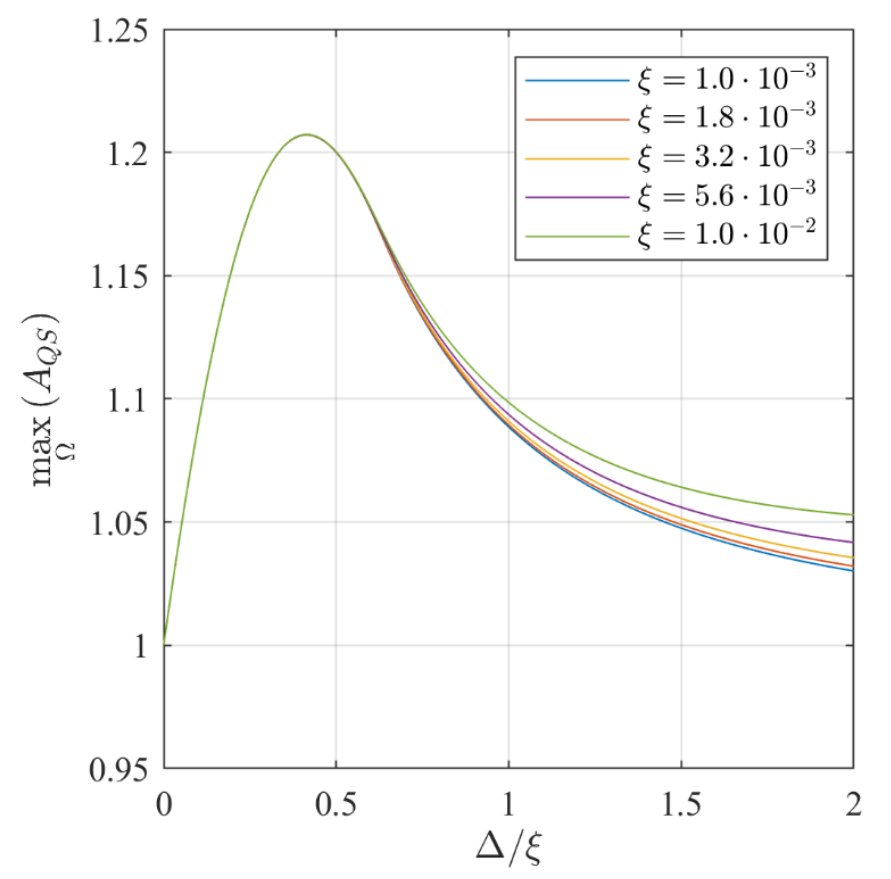


Figure 6

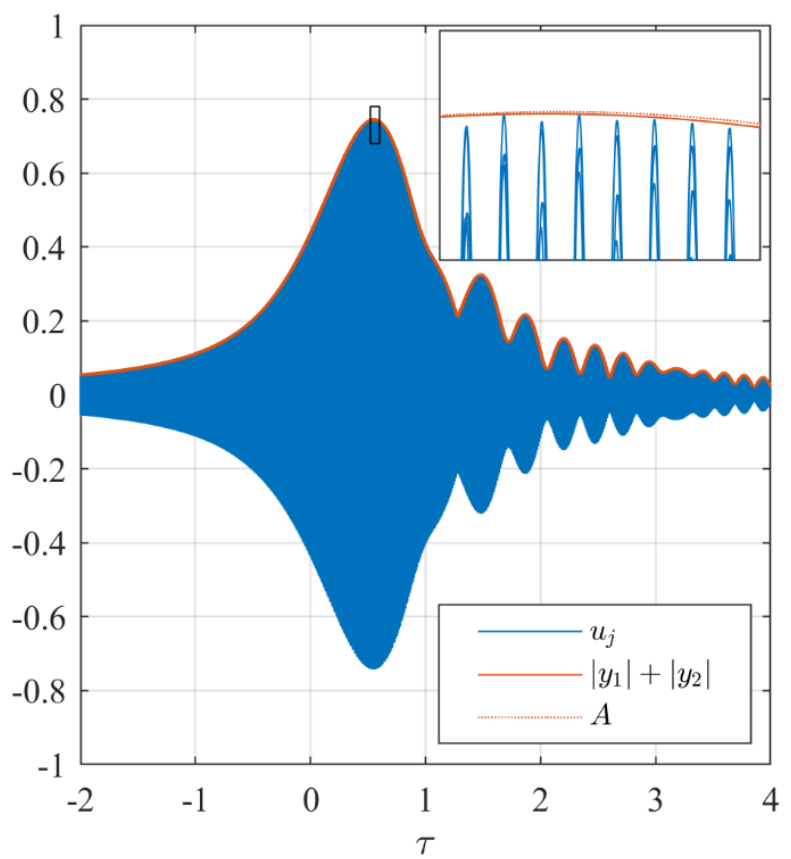


Figure 7

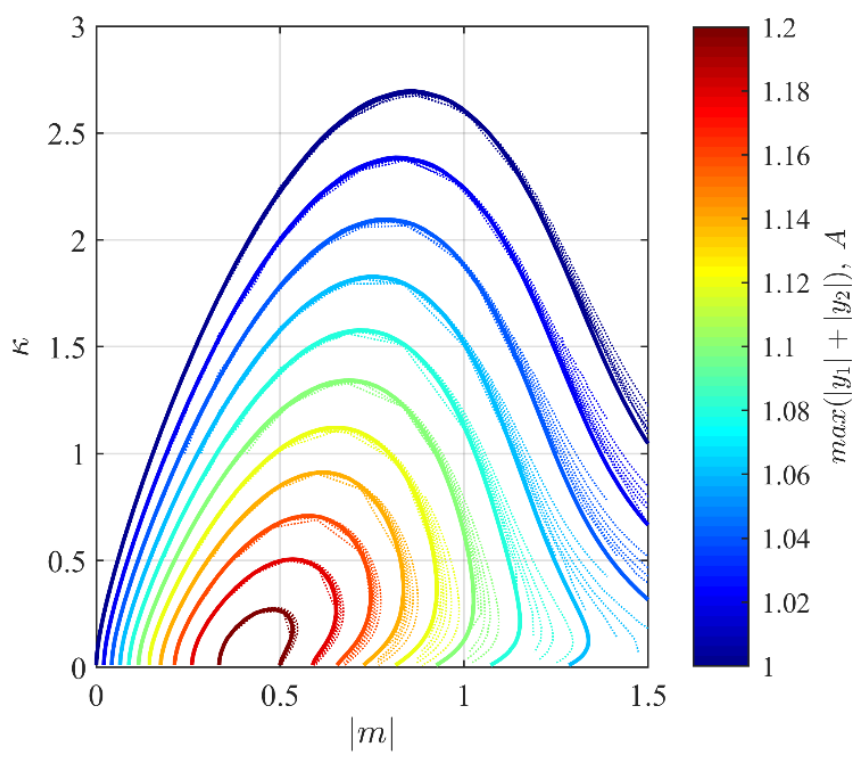


Figure 8

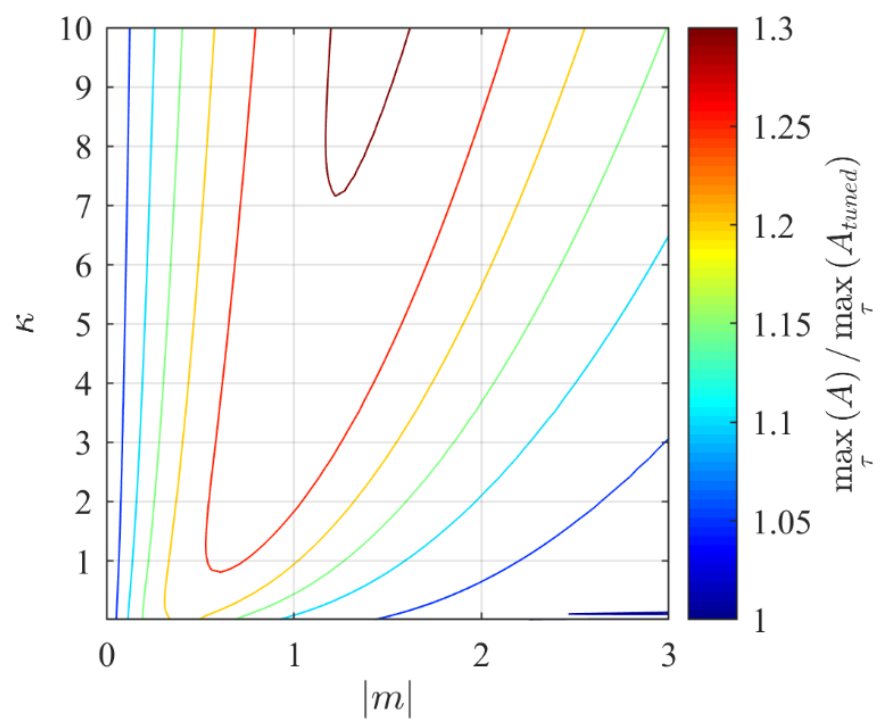


Figure 9

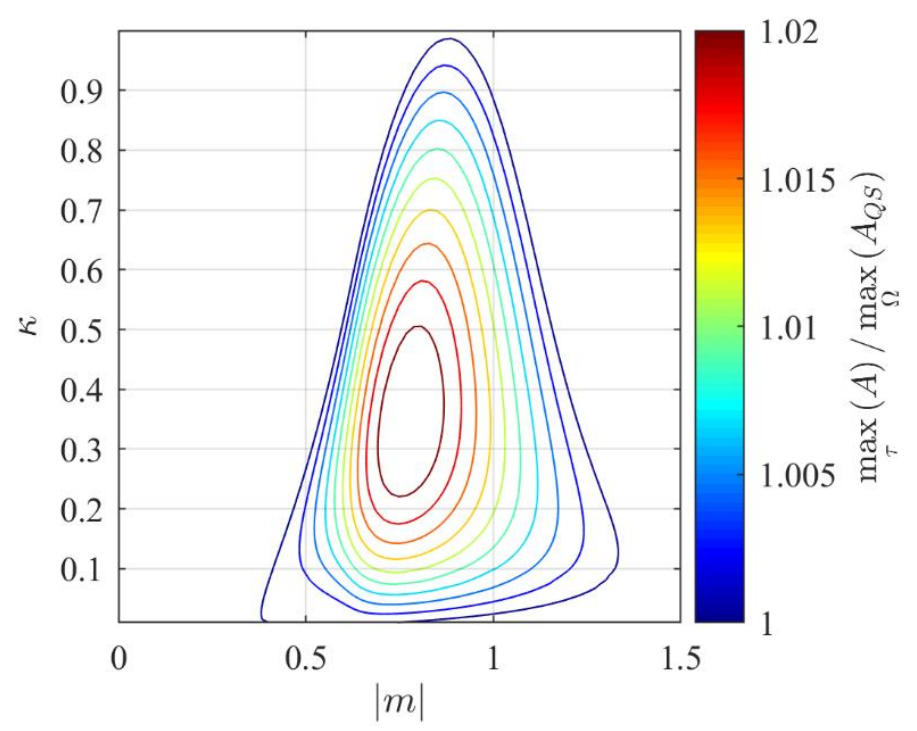

